\title{
INTERPHASE OF A POLYMER AT A SOLID INTERFACE
}

H.J. Butt ${ }^{2}$, H. Duran ${ }^{2,5}$, W. Egger ${ }^{6}$, F. Faupel ${ }^{1}$, V. Harmandaris ${ }^{2,3}$, S. Harms ${ }^{1}$, K. Johnston ${ }^{* 2,4}$, K. Kremer ${ }^{2}$, F.Y. Lin ${ }^{2}$, L. Lue ${ }^{4}$, C. Ohrt ${ }^{1}$, K. Raetzke ${ }^{1}$, L. Ravelli ${ }^{6}$, W. Steffen ${ }^{* 2}$, S.D.B. Vianna ${ }^{2}$

${ }^{1}$ University of Kiel, Institute for Materials Science, Chair for Multicomponent Materials, Kaiserstr. 2, D-24143 Kiel, Germany

${ }^{2}$ Max Planck Institute for Polymer Research, P.O. Box 3148, 55128 Mainz, Germany

${ }^{3}$ University of Crete, Department of Applied Mathematics, Heraklion, Greece

${ }^{4}$ University of Strathclyde, Department of Chemical and Process Engineering, 75 Montrose Street, Glasgow G1 1XJ, United Kingdom

${ }^{5}$ TOBB University of Economics and Technology, Dept. of Mater. Sci \& Nanotechnol. Eng. 06560, Ankara, Turkey

6 Institut für Angewandte Physik und Messtechnik LRT2, Fakultät für Luft- und Raumfahrttechnik, Universität der Bundeswehr München, Werner Heisenberg-Weg 39, 85577 Neubiberg, Germany

ABSTRACT: Atomistic molecular dynamics simulations of a chemically realistic model of atactic short-chain polystyrene between gold surfaces (111) and Positron Annihilation Lifetime Spectroscopy experiments on similar polystyrene thin films on gold were performed. Results from both approaches show that the free volume voids in the film have a slightly smaller average size than in bulk polystyrene. In agreement to that the existence of an interphase of higher density at the polymer-solid substrate interface is shown both by the simulation as well as in the experiment. The average shape of the voids is similar in the bulk and the film. 


\section{INTRODUCTION}

Polymer-solid interfaces are at the center of intensive research due to their importance in polymer-coatings, hybrid materials, lubrication, adhesion, etc. The polymer properties close to this interface are of paramount importance for the performance of these composite systems. From the viewpoint of fundamental science, the confinement imposed by a solid substrate on the polymer should affect the polymer's glass transition temperature, and this has led to a number of studies.

Various properties of polymers confined in thin films, be it supported or free standing, differ from their bulk properties. In the case of supported thin films, an interphase between the substrate and the bulk phase of the polymer is postulated, and the width of this interphase layer has been the focus of many studies. Confinement effects on polystyrene thin films and their implications for the polymer glassy dynamics were studied by molecular dynamics (MD) simulation [1]. For freestanding polystyrene films effects on the glass transition in a boundary layer towards the gas phase a lower glass transition temperature was found [2]. On a completely smooth, structureless, solid substrate modeled by a truncated 9-3 Lennard-Jones potential three layers with different density and glass transition behavior - substrate, middle, surface - have been shown in MD simulations [3]. MD simulations of polystyrene on gold [4, 5], polybutadiene on graphite [6] and polyethylene on graphite [7] have shown the existence of a higher-density interface layer of a width of about $2 \mathrm{~nm}$. The dynamical properties of polymers near an interface have also been probed using MD simulations [4,6,8]. Experimental work about polymers at the interface claim the existence of e.g. a "dead layer" or "Guiselin brushes" [9] of a few nanometer at the interface as permanently attached polymer chains where even washing with a good solvent cannot remove this layer again. Using gold nanoparticles as markers in a X-ray Photon Correlation (XPCS) experiment [10], an irreversibly adsorbed layer with a surface reduced viscosity (compared to bulk viscosity) layer altering the dynamics up to a distance of approximately $20 \mathrm{~nm}$ from the substrate was postulated as interpretation of their results. With the recently developed technique Resonance 
Enhanced Dynamic Light Scattering (REDLS), experiments on polybutadiene [11] showed a slowing-down in dynamics, and hence an increase in viscosity going to films below $25 \mathrm{~nm}$ in thickness. This is explained in terms of a three layer model of which one of the layers is a layer at the gold interface with strongly slowed down dynamics. These results are in concord with the computer simulations [6] on polybutadiene. In all cases the dynamics at the interface is found to be significantly slower than in the bulk. On the other hand for the case of polystyrene, there exists extensive work with dielectric spectroscopy [12] that finds no indication of a change from bulk behavior in the segmental dynamics. The smallest film thickness reported in these experiments was $5 \mathrm{~nm}$. The dynamics of a single polymer chain was studied with dielectric spectroscopy [13] and only bulk behavior of the dynamics of the polymer segments was found. These results contradict the existence of a "dead layer" and leave the question why a method measuring directly the dynamics of polymer segments does not see what more indirect methods as depicted above see - a slowdown of dynamics in the proximity of the solid interface.

In this paper we report a joint study of thin polystyrene films at a gold interface comparing experimental and simulation results. For the experimental part, positron annihilation lifetime spectroscopy (PALS) was used to investigate the free volume as function of distance from polymer film surface to the substrate. Atomistic molecular dynamics simulations were performed and the density and free volume (unoccupied space), approaching a solid substrate for ultrathin films of polystyrene on gold, were analyzed. The polymer samples used in the experiment and simulation were similar in molecular weight. The temperature range to be used in PALS was below $\mathrm{T}_{\mathrm{g}}$ as significantly above $\mathrm{T}_{\mathrm{g}}$ holes in the polymer decay on the measurement time scale while in simulations due to finite computing time only temperatures well above $\mathrm{T}_{\mathrm{g}}$ can be studied. Nevertheless, the difference between the polystyrene bulk and film can be compared. 


\section{EXPERIMENTAL}

\section{Sample Preparation}

All polystyrene (PS) specimens were produced at the Max Planck Institute for Polymer Research by anionic polymerization. The specimens have a polydispersity $\mathrm{M}_{\mathrm{w}} / \mathrm{M}_{\mathrm{n}}$ better than 1.2 in all cases. For PALS we used oligomeric atactic polystyrene of $\mathrm{M}_{\mathrm{w}}=400 \mathrm{~g} / \mathrm{mol}$ (4mer) and $700 \mathrm{~g} / \mathrm{mol}$ (7mer).

All samples were deposited on ultra-flat, polished N-LaSF9 glass substrates (Hellma) of thickness $\mathrm{d}=1.5 \mathrm{~mm}$. In a first step, after cleaning the substrates from residual dust, $1 \mathrm{~nm}$ of chromium as adhesion promoter followed by $100 \mathrm{~nm}$ of Au were deposited by thermal evaporation onto the substrates. To produce the polymer films we drop casted PS/Toluene solutions (10 \%w/w) onto the $\mathrm{Au}$ on the substrates and annealed the films subsequently at $50 \mathrm{~K}$ above $\mathrm{T}_{\mathrm{g}}$ for at least $24 \mathrm{~h}$ to remove residual solvent. We produced two sets of samples with thicknesses of $d=250 \mathrm{~nm}$ (400 $\mathrm{g} / \mathrm{mol})$ and $\mathrm{d}=350 \mathrm{~nm}(700 \mathrm{~g} / \mathrm{mol})$. The film thickness was confirmed by confocal microscopy and by profilometer (Tencor P-10). All samples were further annealed at $\mathrm{T}_{\mathrm{g}}+40 \mathrm{~K}$ for $36 \mathrm{~h}$ under vacuum. We established by profilometry that in the center of the glass slide an area bigger than the beam size for PALS had a constant thickness.

\section{Samples measured with PALS:}

The following samples were used for the positron beam measurements. The samples "AU” and "PS700" were used as reference to separate the influence of the Au layer on the results and interphase width, respectively. With the other two samples the influence of molecular weight and film thickness could be identified and separated. 
Table 1: Sample names and sample characteristic for the PALS experiment. Glass is N-LaSF9, the thickness of the CR layer is $1 \mathrm{~nm}$ and the thickness of the Au layer $100 \mathrm{~nm}$ in all relevant cases.

\begin{tabular}{|c|c|c|c|}
\hline Sample name & layers & Mw PS [g/mol] & h [nm] PS film \\
\hline "AU" & Glass + Cr + Au & - & - \\
\hline "PS700" & Glass + PS & 700 & 350 \\
\hline "AU PS700" & Glass + Cr + Au + PS & 700 & 350 \\
\hline "AU PS400" & Glass + Cr + Au + PS & 400 & 250 \\
\hline
\end{tabular}

\section{PALS}

Positron annihilation lifetime spectroscopy is a well-accepted technique for determining free volume in polymers and composites $[14,15,16,17,18,19]$. This technique is based on probing the electron density distribution, which is related to the unoccupied regions (free volume) of the polymer. Thus, positrons injected into the polymer, thermalize and either annihilate directly with electrons from the sample ("free positron, lifetime $\tau_{2} \sim 400-600 \mathrm{ps} "$ ) or form eventually a bound state with electrons (i.e. positronium) localized in free volume cavities or voids. The para state (spin of electron and positron antiparallel) decays immediately ( $\tau_{1}=125 \mathrm{ps}$ ) whereas the ortho state (spin of electron and positron parallel, $\tau_{3}$ ) cannot decay due to spin conservation. This ortho positronium can be used as probe for the cavity size: the larger the cavity the smaller the corresponding overlap of the square of the o-Ps wave function and cavity wall. The relative probabilities of these three decay modes are usually termed intensities and are related to the positronium formation probability.

A well accepted quantum mechanical model makes this quantitative [20]

$$
\tau_{o-P s}=\lambda_{0}^{-1}\left(1-\frac{R_{h}}{R_{h}+\delta R}+\frac{1}{2 \pi} \sin \frac{2 \pi R_{h}}{R_{h}+\delta R}\right)^{-1}
$$


where $\lambda_{0}$ is the spin averaged decay rate in the electron layer at the edge of the potential well, $\delta \mathrm{R}=0.166 \mathrm{~nm}$ is the thickness of the electron layer, which has been determined by using materials with a well-known pore size [21] and $R_{h}$ is the average free volume hole radius. In summary, PALS is a suitable tool to determine the average hole size of free volume in polymeric materials. In previous works some of the present authors have shown that PALS results in bulk polymers qualitatively fit to simulations [22] and that these simulations can also be evaluated with respect to size distribution and shape of the free volume [23].

For the present investigation, it is sufficient to assume that the decrease in o-Ps lifetime $\tau_{3}$ is correlated with the decrease in average hole size.

Conventional tracer-based positron sources emit positrons with very high energy, thus up to $1 \mathrm{~mm}$ of polymer is necessary for complete absorption. For depth resolved experiments with planar samples, an energy tunable positron lifetime beam is necessary - like the one available at the FRM II in Munich [24], which is now routinely used for thin film polymeric samples [17, 25]. Here the distribution of the positrons as function of distance to the surface for different energies has to be taken into account. In a homogeneous material, this probability distribution $\mathrm{P}(\mathrm{z}, \mathrm{E})$, where $\mathrm{z}$ is the position and $\mathrm{E}$ is the implantation energy, is analytically solvable and follows the so-called Makhov profile.

$$
P_{i}(z, E)=\frac{m z^{m-1}}{z_{0}^{m}(E)} \exp \left[-\left(\frac{z-\delta z_{i}}{z_{0}(E)}\right)^{m}\right]
$$

with

$$
z_{0}(E)=z_{1 / 2}(E)(\ln 2)^{-1 / m}
$$


where $z_{1 / 2}$ is the median implantation depth, $E$ is the energy of the incoming positrons, $A$ is a constant factor, $\rho$ is the density of the material and $m$ and $n$ are numerical factors. For practical applications, the shortened form with

$$
z_{1 / 2}(E)=\frac{A}{\rho} E^{n}
$$

is used.

Unfortunately, the implantation profile widens with increasing implantation energy, and consequently the depth resolution decreases. Qualitatively, the uncertainty, $\Delta \mathrm{z}$, is given by

$$
\Delta z \sim 0.5 * z_{1 / 2}(E)
$$

[26]. Although these Makhov profiles might only be approximate, they are frequently used [27], in particular for polymers, and have also been verified by computer simulations $[28,29]$. One of the most common computer programs for simulation of high energetic particles with solid matter is GEANT4 [30], originally developed for detectors in high-energy physics research.

GEANT4 has also been applied to layered metallic systems and has been shown to be very useful [28], as the back reflection on layers with higher density can be taken into account. Some of the present authors have already performed reference PALS beam measurements on layered polymersolid systems, namely Teflon AF on a Si substrate, where no such problems with back reflection of positrons at the substrate occurs. In these studies it was shown that a clear interphase with a lower free volume exists for spin coated polymer films. However, for the present samples, problems due to backscattering are to be expected due to the higher density and atomic number $\mathrm{Z}$ of the Au layer underneath the polymer.

\section{PALS beam conditions}

Positron annihilation lifetime spectroscopy was performed at the PLEPS of the NEPOMUC at the FRM II in Munich [24] at a vacuum of $10^{-6}$ mbar with a beam spot diameter of $\sim 2 \mathrm{~mm}$ at 
implantation energies from $1 \mathrm{keV}$ to $16 \mathrm{keV}$ with counting times of $500 \mathrm{~s}$ per point at a typical peak to background ratio of $5 \times 10^{3}$ to 1 .

Positron lifetime spectra were treated as usual with the program LT 9.2 Kansy [31], which is particularly suited for results from polymers, as a distribution of lifetimes can be taken into account. Three and four lifetimes were determined, some of them fixed according to known rules (for details see below). The usual corrections [17,20,22] due to the background and the energy dependent resolution function have also been performed.

\section{SIMULATION DETAILS}

A thin polystyrene film of width $10 \mathrm{~nm}$, sandwiched between two gold surfaces, was simulated at $1 \mathrm{~atm}$ and $503 \mathrm{~K}$ for $100 \mathrm{~ns}$ using NpT classical atomistic molecular dynamics as implemented in the GROMACS package version 4.6 [32,33,34]. The film contained 50 chains, each of length 10 monomers. The interaction between the polymer and the surface was obtained from quantum density functional theory calculations $[4,35]$. The simulations are described in more detail in a previous paper [4]. The current work further analyses the interface density and focuses on the structure and distribution of the free volume in the films near the interface, which can be used to understand the PALS observations.

To analyze the free volume distribution in the polystyrene, the simulation cell was divided into a simple orthogonal grid with spacing $\sim 0.05 \mathrm{~nm}$ (the exact spacing depended on the fluctuating box size). Any grid points within a distance $\sigma$ of polystyrene or gold atoms were removed, leaving only those grid points occupying the free volume in the film, referred to as free volume grid point or, in short, voxel (three dimensional analogue to pixel). We note here that all atoms have the same size in this analysis. This network of free volume voxels was divided into clusters, and the voxels were assigned to a cluster if they were closer than a distance, $\mathrm{d}=0.06 \mathrm{~nm}$ (just larger than 
the grid point spacing), from any other voxel in that cluster. Thus we name the free volume entities determined this way from simulations free volume clusters.

To obtain information about the structure of the film, the free volume cluster properties, such as size and shape, were analyzed. The shape of a free volume cluster was analyzed via the gyration tensor and its eigenvalues, denoted $\lambda_{1}, \lambda_{2}$ and $\lambda_{3}$, where $\lambda_{1} \geq \lambda_{2} \geq \lambda_{3}$. A measure of the shape of the free volume cluster is given by the asphericity, A, which is defined as

$$
A=\frac{2 \lambda_{1}-\lambda_{2}-\lambda_{3}}{2\left(\lambda_{1}+\lambda_{2}+\lambda_{3}\right)}
$$

so that a sphere would have $A=0$, an infinitely thin planar free volume cluster would have $A=0.25$, and an infinitely thin cylinder would have $A=1$. The free volume analysis was performed over 10 ns with the configurations separated by 100 ps.

\section{RESULTS AND DISCUSSION}

\section{1) PALS}

The qualitative behavior of the positron data is similar to previous investigations [25]. The o-Ps intensity, being a measure for o-Ps formation probability multiplied by hole concentration, decreases with increasing energy due to the increased implantation of positrons into the non-o-Ps forming substrate. The increase above $10 \mathrm{keV}$ only occurs for the samples with the Au-layer. This Au-layer causes back-reflection of the positrons, leading not only to spurious artifacts in the original annihilation spectra but also to enhanced sensitivity in the interphase region. This speculation could be confirmed by simulations with GEANT4 [36]. The o-Ps lifetime is a measure for the average free hole size, as mentioned above (eq. 1). For energies between 1-4 keV, reflecting mainly annihilation in the bulk of the polymer, the observed o-Ps lifetimes correspond to literature values [15]; for higher energies, where it is clear from the decrease in o-Ps intensity, the o-Ps lifetime also decreases. This decrease in the o-Ps lifetime is only from the 


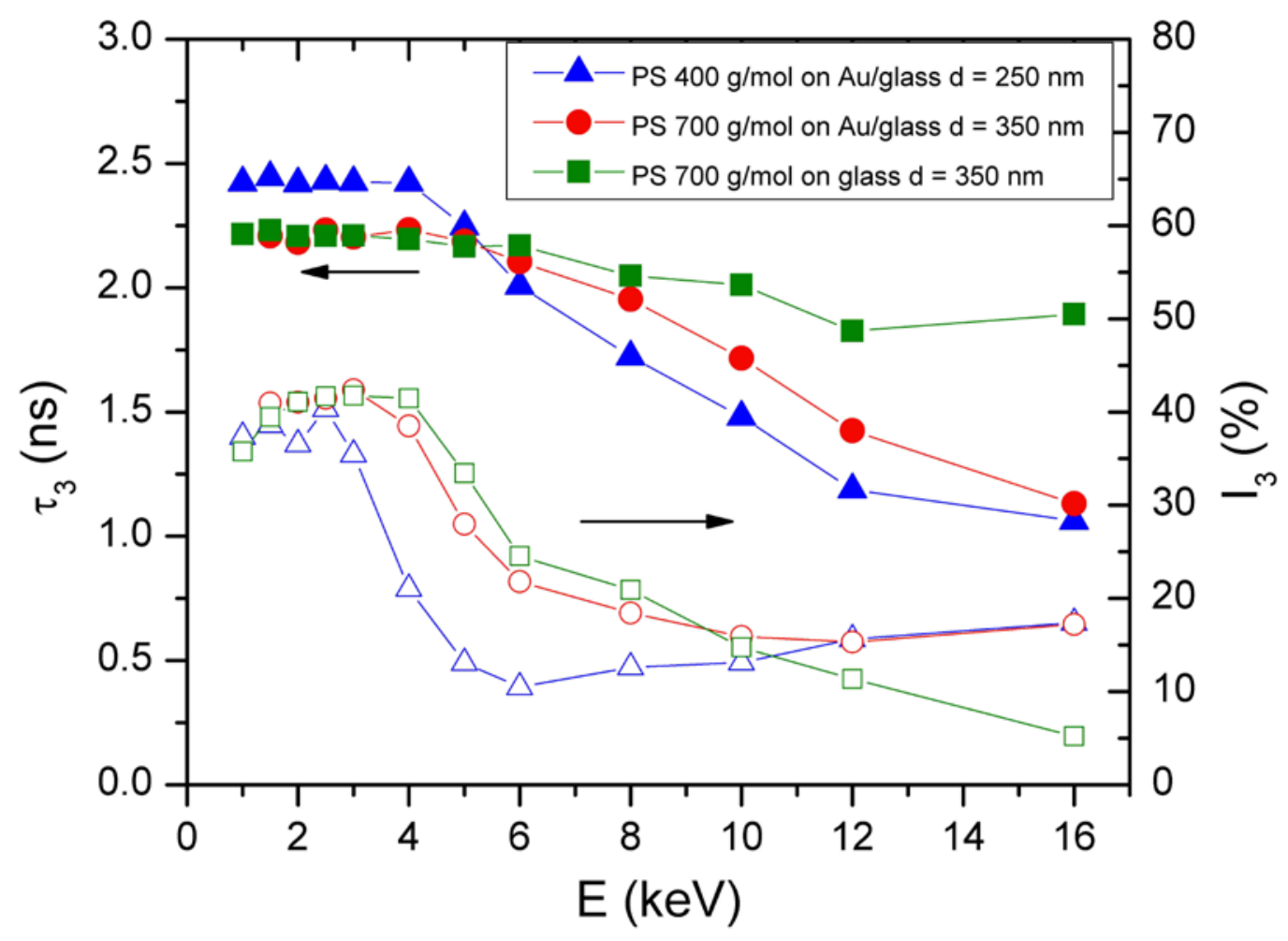

Figure 1: O-Ps lifetime, (filled symbols, left hand scale) and o-Ps intensity (unfilled symbols, right hand scale) as function of implantation energy for different molecular weights and film thickness.

polymer (we also performed a detailed analysis giving similar results but taking into account the contribution of glass substrate). The main problem of an numerical determination of interphase width and reduction in density is the broadening of the implantation profile, when analysis from the polymer side is done. In another paper [37]we analyzed the interphase region between Teflon AF and SiN/Si substrate through a hole in the substrate, thus avoiding broadening effects in the interphase region. A detailed analysis there and comparing these results with [25] showed that the interphase width is of the order of $10 \mathrm{~nm}$. Thus, it is reasonable to assume also for the present 
experiments an interphase width of approx. $10 \mathrm{~nm}$. Thus the average o-Ps lifetime in a polymer layer close to the substrate is lower than in the bulk of the film. As the shape of the holes does not change throughout the film (see below), eq. 1 shows that the average hole size significantly decreases. Quantitative information - also on the corresponding length scale - is not straightforward to obtain, as the density profile is convoluted with the implantation profile and possible diffusional broadening. Qualitatively we can state that the lower limit of resolution of the length scale of the interphase is approximately $2 \mathrm{~nm}$, as we have not seen the native $\mathrm{SiO}_{2}$ layer in previous investigations [25]. On the other hand, for implantation energies of 3-4 keV, corresponding to average depth of $175 \mathrm{~nm}$ - 290nm (from eq. 4), bulk behavior is seen, therefore, the interphase width must be somewhat smaller than this. Thus, a more atomistic view with the help of simulations is necessary.

The advantage of simulation is that it can show details that are inaccessible to experiment. However, the simulations are only as accurate as the force fields they used and it is important to validate the simulations using experimental results.

\section{2) Simulation results}

The simulation has investigated several issues: a) the density profile dependence on resolution, b) the dependence of the free volume on the atomic radii and c) the shape of the free volume clusters.

\section{a) Variation of density profile with resolution}

The polystyrene film exhibits high-density oscillations within $1.5 \mathrm{~nm}$ of the gold surface, and in this region the dynamics of the film is reduced compared to bulk dynamics, as previously

observed [5]. However, in the previous work, the binning used to analyze the density has much greater resolution than can be achieved by the PALS technique. For this reason, the atomic mass density was re-analyzed using larger bins, and the density profiles for different resolutions (bin 
widths, w) were calculated. To obtain smoother curves, rolling bins were used. These density profiles are shown in Figure 2.

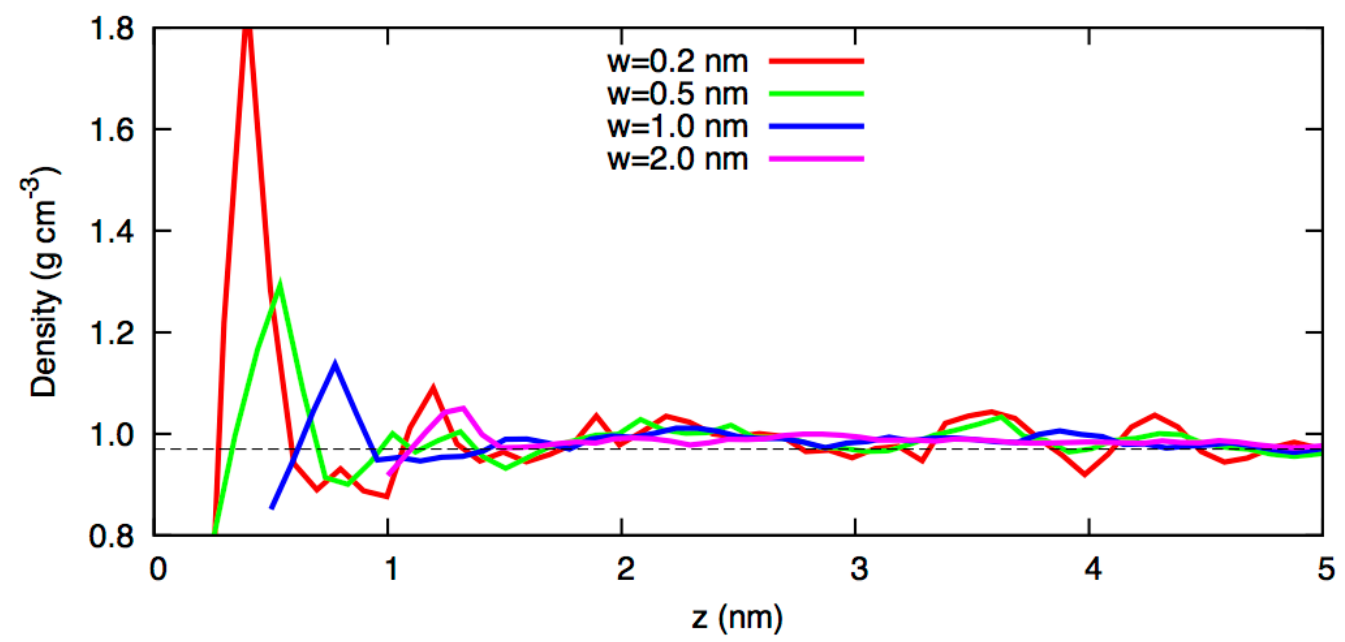

Figure 2: The density profile of the polystyrene film as a function of distance from the surface, $z$. The different lines correspond to different bin widths, $w$, as indicated in the legend. The horizontal black line corresponds to the density of bulk polystyrene.

As expected the high-density peak becomes smeared out as the resolution is decreased (w increased) and for $\mathrm{w}=1.0 \mathrm{~nm}$, the peak is approximately $15 \%$ larger than the bulk value.

b) Size and shape dependence of the free volume on atomic radii

A snapshot of the polymer film and the corresponding free volume clusters is shown in Fig. 3. 


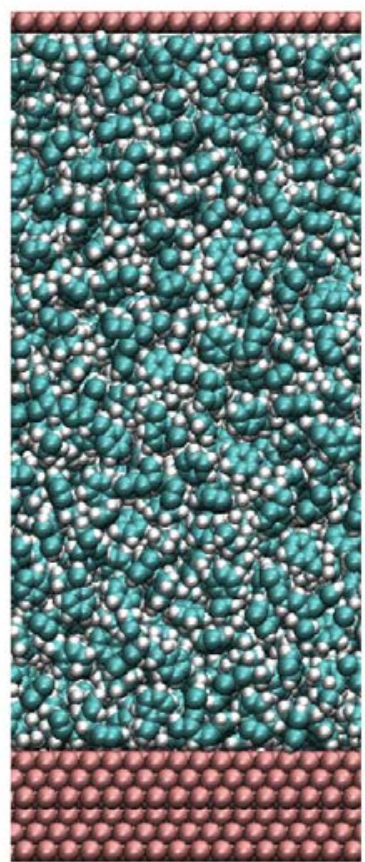

(a)

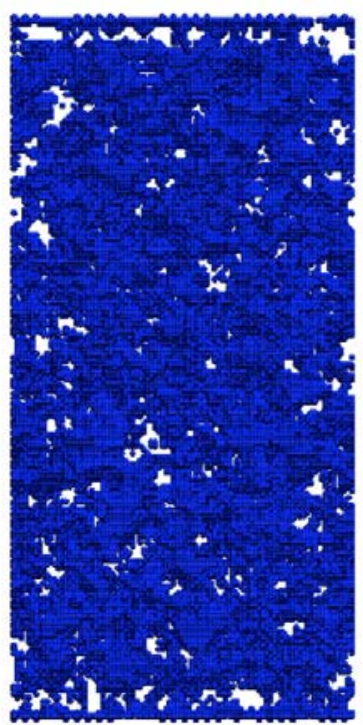

(b)

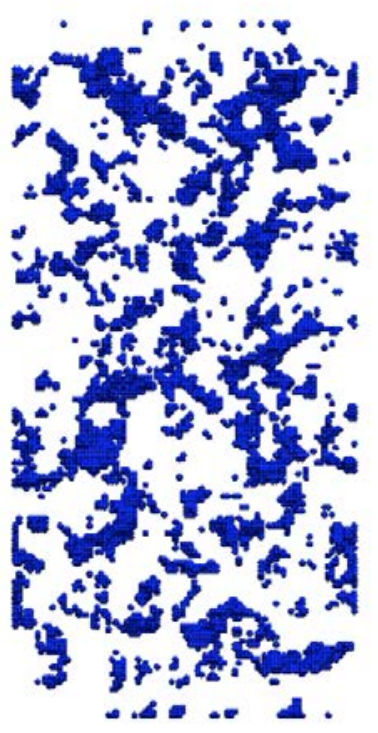

(c)

Figure 3: (a) Snapshot of the $10 \mathrm{~nm}$ film of polystyrene confined between gold surfaces. On the right are the corresponding free volume voxels for (b) $\sigma=0.20 \mathrm{~nm}$ and (c) $\sigma=0.25 \mathrm{~nm}$, respectively.

Obviously, the free volume voxel density depends on the value of the cut-off distance $\sigma$ so that for large values of $\sigma$, the free volume voxel density is small, and for small $\sigma$, the density is large, as can be seen in Fig. 3. The average density can be compared to the average bulk density for each cut-off value. The free volume fraction was calculated by dividing the number of free volume voxels by the number of grid points in the total film volume. The volume fraction of the free volume voxels in the systems has been plotted in Figure 4(a) together with the theoretical prediction for the free volume fraction $\Phi$ of random penetrating spheres in a box given by [38]:

$$
\Phi=\left(1-\rho \frac{4 \pi \sigma^{3}}{3 N}\right)^{N}
$$


where $\mathrm{N}$ is the number of particles and $\rho=\mathrm{N} / \mathrm{V}$ is the number density where $\mathrm{V}$ is the volume of a sphere of radius $\sigma$. In the continuum limit this becomes

$$
\Phi=\lim _{N \rightarrow \infty} \Phi=\exp \left(-\rho \frac{4 \pi \sigma^{3}}{3 N}\right)
$$

The simulation results for bulk polystyrene deviate slightly from the theory for cutoff radii of 0.1 $\mathrm{nm}$ to $0.25 \mathrm{~nm}$. We believe that this is due to the correlation between the polymer atoms in the simulations (i.e. the atoms are not randomly arranged) since the hydrogen atoms are situated close to the carbon atoms and the carbon atoms in a chain have a preferred bond distance. This correlation hypothesis is supported by the fact that both bulk and film deviate from the curve by a similar amount and have a slightly higher volume fraction that the theoretical prediction.

To make a comparison with experiment we are interested in the ratio between the free volume fractions of the film and the bulk, which can be obtained using eq. 8 for bulk PS with average density $\rho_{\mathrm{b}}$ and for the PS film with average density $\rho_{\mathrm{f}}$. The free volume fraction ratio is then given by

$$
\frac{\Phi_{f}}{\Phi_{b}}=\exp \left(-\left(\rho_{f}-\rho_{b}\right) \frac{4 \pi \sigma^{3}}{3}\right)
$$

and depends on the cutoff radius $\sigma$. The ratio between the film and bulk free volume voxel densities is shown in Fig. 4(b). The ratio of the free volume fraction in the film and bulk shows a clear decrease as the cutoff increases. This can be quantified by fitting equation 8 to the bulk and film data, using $\rho_{f}$ and $\rho_{b}$ as fitting parameters, and then calculating $\Phi_{f} / \Phi_{b}$. This curve also decreases with increasing cutoff, as shown in Figure 4b, although the decrease is much slower than that using the raw data and this difference can be further understood by considering the fluctuations in free volume. As $\sigma$ increases, the free volume decreases, and the relative distribution of free volume for different snapshots is much larger. The error bars show the standard deviation 
of the film free volumes for different snapshots and indicate that only the data from cutoffs up to about $0.20 \mathrm{~nm}$ is reliable. The standard deviation of the bulk sample is not included in the bars.

In addition to the average film data the data from layers parallel to the surface are shown. The data are averaged over both gold surfaces. Layers 1-4 are of width $1.0 \mathrm{~nm}$, but Layer 5 is smaller, approximately $0.9 \mathrm{~nm}$, being the central region, and its width varies with the box size. Layer 1 is closest to the gold surface and shows a smaller free volume than the film average, which is due to the presence of the gold surface and the high PS density. This decrease in free volume voxels can be seen in the snapshots of the free volume voxels in Fig. 3(b) and (c) and is consistent with the high density layer shown in Fig. 2. The second layer has a smaller free volume fraction ratio, which is due more free volume voxel particles in this layer compared to the film average. Similarly layers 3, 4 and 5 have a slightly higher free volume voxel particle density than the film average. Within error bars, layers 2-5 have a similar density to bulk but beyond $\sigma=0.20 \mathrm{~nm}$ the ratio between the layer free volume to bulk free volume is within the error bars.

\section{c) Free volume voxel cluster size and shape}

To understand more about the bulk and film structures, the asphericities of the free volume clusters have been analysed. Information about the number density of the clusters is given in supporting information. It can be seen in Figure 5 that for $\sigma=0.20 \mathrm{~nm}$ there are several very large clusters due to the formation of free volume voxel networks. These large clusters are affected by the periodicity of the box and have anomalous asphericities. For bulk PS these continuous networks have low asphericities between 0 and 0.25 , and correspond to planar networks. A similar situation can be seen for the PS film, where large free volume clusters exhibit asphericities of around 0.2 or 0.8 . Therefore, to obtain a realistic cluster shape analysis $\sigma$ should be larger than $0.20 \mathrm{~nm}$. 

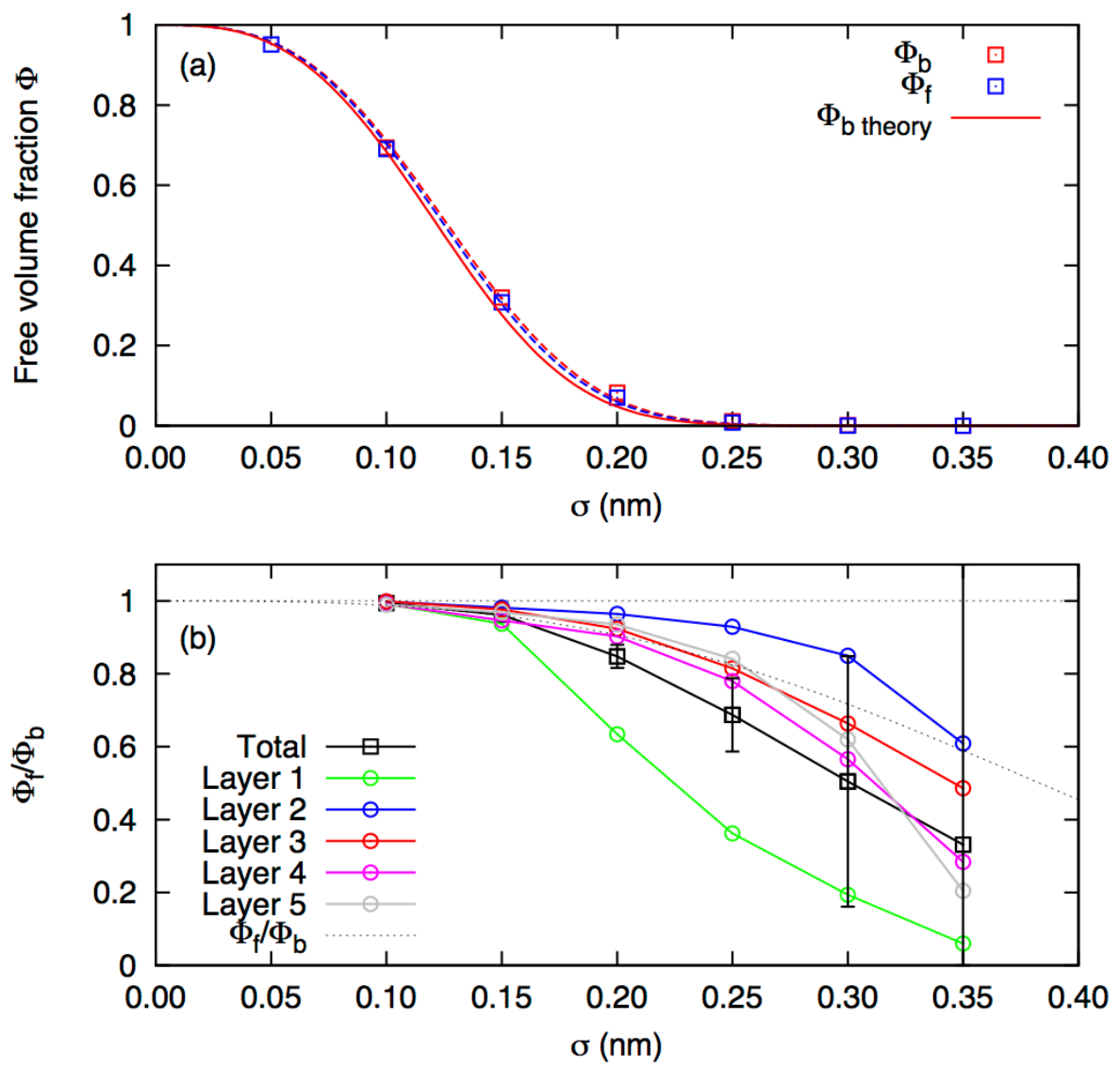

Figure 4: (a) Free volume fraction for bulk PS and the thin film of PS on Au (symbols). The theoretically predicted volume fraction as a function of cut-off radius using the bulk density is shown as a solid line. The dashed lines are fits of equation 8 to the bulk and film data where the density is treated as a fitting parameter. (b) Ratio of the free volume fractions for the thin film and bulk polystyrene. Total refers to the whole film and the layers are slices of the film parallel to the interface, with Layer 1 being closest to the gold. The black dashed line comes from the fitted $\Phi_{\mathrm{b}}$ and $\Phi_{\mathrm{f}}$ in (a). 
(a) Bulk

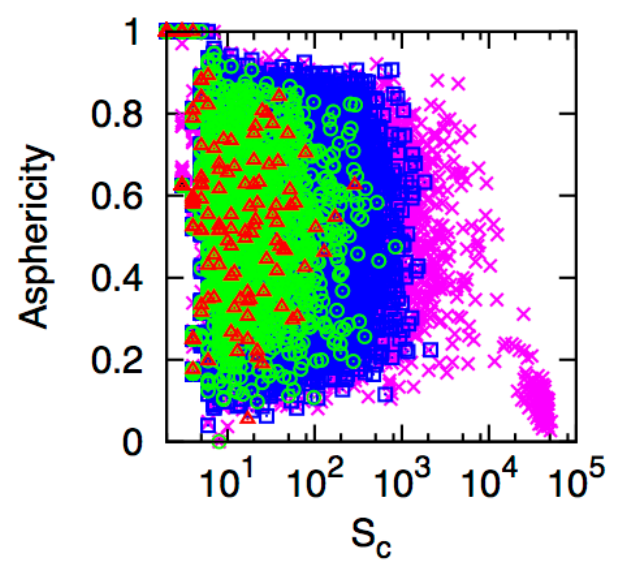

(b) Film

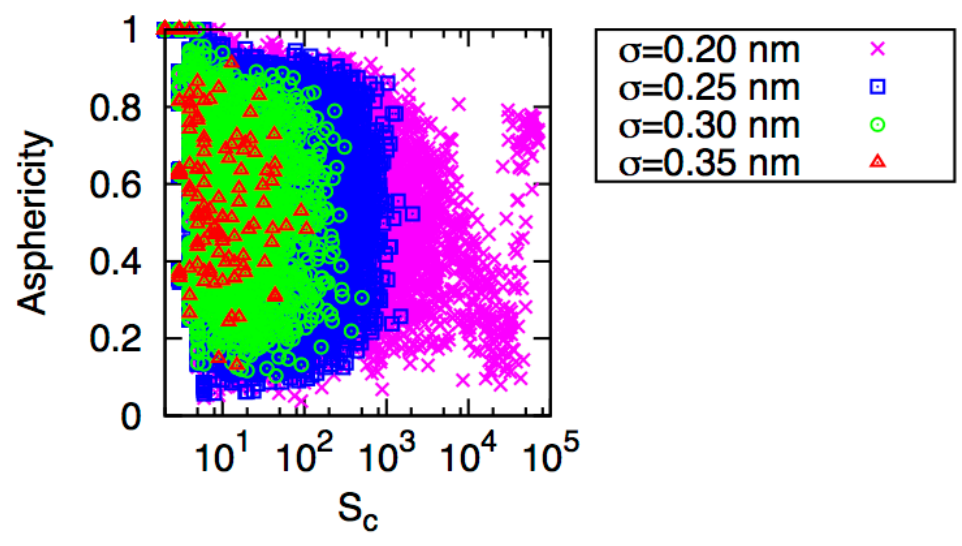

Figure 5: Cluster asphericity vs. cluster size for (a) bulk PS and (b) the PS film. For $\sigma=0.20$ nm large clusters have an anomalous asphericity distribution due to the formation of continuous networks.

For small free volume clusters of less than 10 voxels there is an issue with discretisation of the cluster shape due to the grid, and, hence, the average asphericity of these clusters is larger than for larger clusters. For example, all 2-voxel free volume clusters have an asphericity of 1 . The average asphericity of free volume clusters of a particular size decreases up to a size of around 10, when the average becomes constant, as shown in Figure 6. For this reason, clusters with less than or equal to 10 free volume voxels have been omitted from the cluster analysis.

The mean free volume cluster size and mean asphericity (equ. 6) over all clusters of more than 10 voxels for bulk and film and different cutoff values is shown in Table 2. The mean asphericity of around 0.50-0.53 for bulk PS is in good agreement with a previous study of PS that found an average asphericity of 0.5 [39]. For cutoffs of greater than $0.20 \mathrm{~nm}$, the average size of the free volume clusters is larger in the bulk than in the film, in agreement with the findings of the PALS measurements.

Finally, the distribution of free volume clusters with asphericity for bulk PS and the PS film is analyzed. To obtain the best compromise between statistical accuracy and free volume cluster 
(a) Bulk

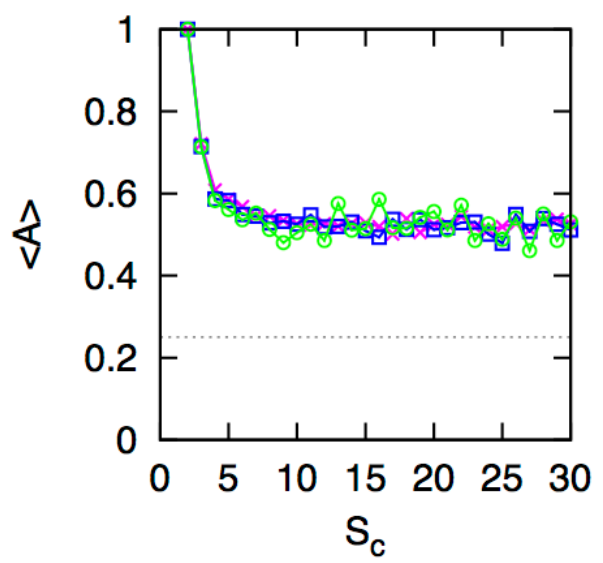

(b) Film

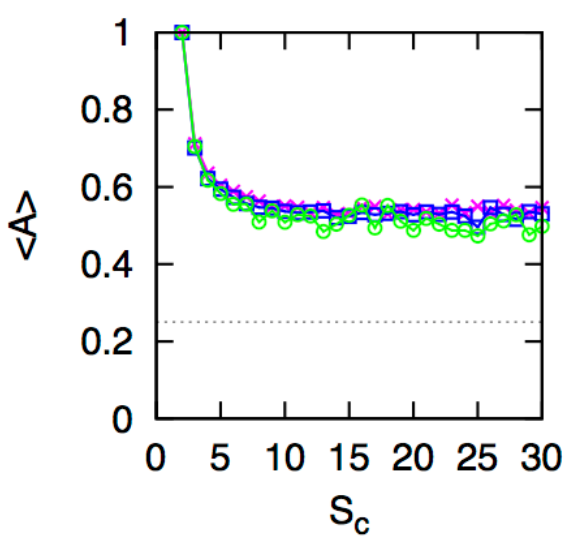

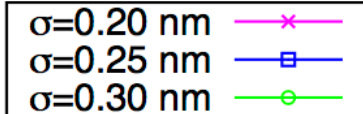

Figure 6: The dependence of mean asphericity on cluster size for (a) bulk and (b) film. The dashed line corresponds to $A=0.25$, which is a planar cluster. $A=1$ corresponds to an infinitely thin cylinder (line).

Table 2: Mean cluster size $<$ Sc $>$ and mean cluster asphericity $<A>$ for different values of the cutoff $\sigma$.

\begin{tabular}{|c|c|c|c|c|}
\hline \multirow{2}{*}{} & \multicolumn{2}{|c|}{ BULK } & \multicolumn{2}{c|}{ FILM } \\
\hline$\sigma[\mathrm{nm}]$ & $<\mathrm{Sc}>$ & $<\mathrm{A}>$ & $<$ Sc $>$ & $<\mathrm{A}>$ \\
\hline 0.25 & 74.4 & 0.53 & 62.7 & 0.53 \\
\hline 0.30 & 44.3 & 0.52 & 36.4 & 0.51 \\
\hline 0.35 & 36.4 & 0.50 & 25.4 & 0.52 \\
\hline
\end{tabular}

analysis a value of $\sigma=0.25 \mathrm{~nm}$ was chosen. The frequency of free volume clusters with a given asphericity for the bulk and film is shown in Fig. 7. There is no clear dependence of the shape distribution on the size of the cluster. It was also found that the average asphericity of the free volume clusters does not depend on the distance from the interface. 
(a) Bulk

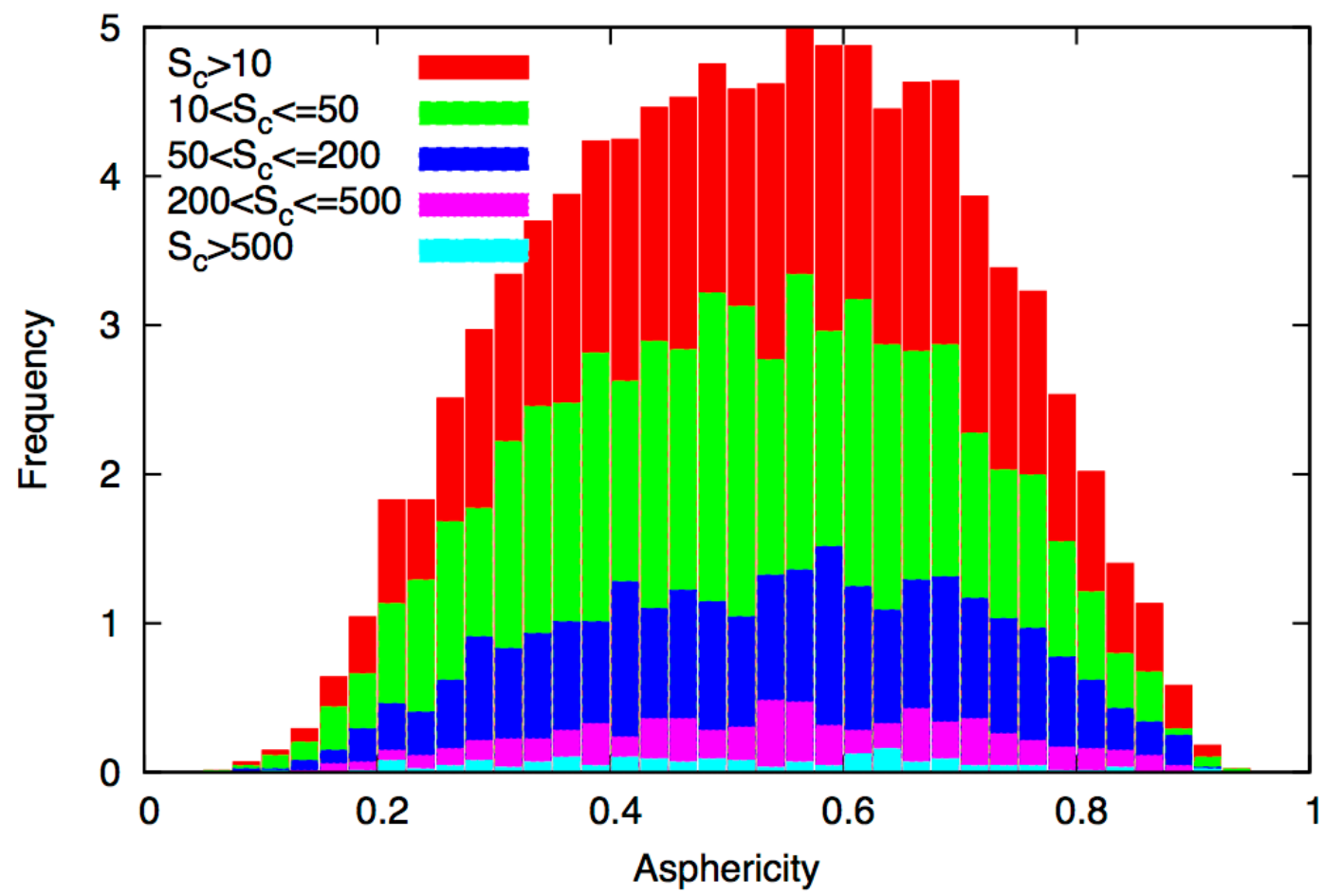

(b) Film

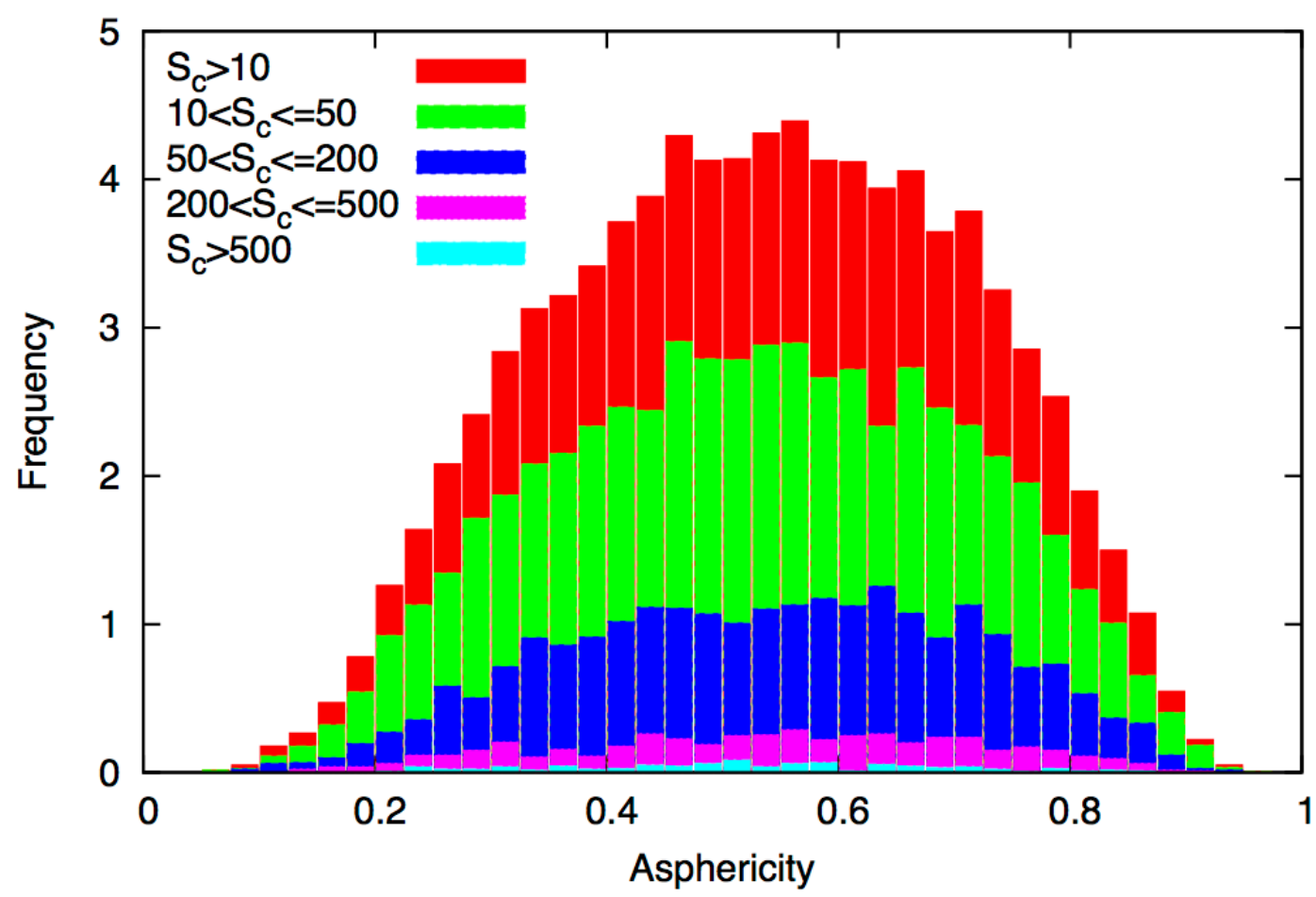

Figure 7: Distribution of clusters with asphericity using $\sigma=0.25 \mathrm{~nm}$ for (a) bulk and (b) film. 


\section{CONCLUSIONS}

The structural properties of polystyrene close to a gold interface have been investigated using both atomistic molecular dynamics simulations and positron annihilation lifetime spectroscopy.

The results of simulations at high temperatures show that the density is affected only about $1 \mathrm{~nm}$ from the gold interface and the average density across a $10 \mathrm{~nm}$ film is only slightly higher than the bulk density. However, an analysis of the free volume fractions in bulk and film, shows that the ratio of free volume fractions can vary strongly with particle size, even for a small difference in density. An analysis of the free volume clusters shows that the shape distribution of the free volume clusters is similar in the bulk and the film and that the distribution of free volume cluster shapes is size-independent. In agreement with experiment, the size of the free volume clusters in bulk polystyrene was found to be larger than the average size of the free volume clusters in the film.

The results from the PALS experiments at low temperatures close to $T_{g}$ show an increase of density towards the gold interface.

Qualitatively the two methods, simulation and PALS, come to the same conclusion of a more dense layer close to the gold interface via the monitoring of the free volume in both methods. It is not appropriate to make a quantitative statement since the two methods operate at different temperatures due to their own respective limitations.

\section{ACKNOWLEDGEMENT}

Financial support by DFG within the SPP 1369 "Polymer-Solid Contacts: Interfaces and Interphases (Projects Ra 796/5 and BU 1556/31-1) is gratefully acknowledged. 


\section{CORRESPONDING AUTHORS}

*(K.J.): Telephone: $\quad+44 \quad(0) 141548$ 4084. Fax: $+44 \quad(0) 141548$ 2539. $\quad$ E- mail: karen.johnston@strath.ac.uk, *'(W.S.): Telephone: +49 (0)6131 379116. Fax: +49 (0)6131 379100. E- mail: steffen@mpip-mainz.mpg.de.

Supporting Information Available: Description. This material is available free of charge via the Internet at http://pubs.acs.org.

\section{REFERENCES}

\footnotetext{
${ }^{1}$ Lyulin, A. V.; Hudzinskyy, D.; Janiaud, E.; Chateauminois, A. Journal of Non-Crystalline Solids 2011, 357, (2), 567-574.

${ }^{2}$ Baljon, A. R. C.; Williams, S.; Balabaev, N. K.; Paans, F.; Hudzinskyy, D.; Lyulin, A. V. Journal of Polymer Science Part B: Polymer Physics 2010, 48, (11), 1160-1167.

(3) Hudzinskyy, D.; Lyulin, A. V.; Baljon, A. R. C.; Balabaev, N. K.; Michels, M. A. J. Macromolecules 2011, 44, (7), 2299-2310.

(4) Johnston K., Harmandaris V., Soft Matter 2012, 8, 6320-6332

(5) Johnston K., Harmandaris V., Macromolecules 2013 , 46, 14, 5741-5750.

(6) Yelash, L., Virnau P., K. Binder and W. Paul, Physical Review E 2010 82, 050801(4).
} 
(7) Daoulas, K. Ch., Harmandaris V. and Mavrantzas V. G., Macromolecules 2005, 38, $5780-$ 5795.

(8) Harmandaris V., Daoulas, K. Ch. and Mavrantzas V. G., Macromolecules 2005, 38, 57965809.

(9) Napolitano, S. and Wübbenhorst, M., Nature communications 2011, 2, 260.

(10) Koga, T., Jiang N. et al. Physical Review Letters 2011, 107, 22, 225901(5).

(11) Lin, F.-Y. and Steffen, W., J. Chem. Phys. 2014, 141, 104903

(12) M. Tress, M. Erber, E. U. Mapesa, H. Huth, J. Müller, A. Serghei, C. Schick, K.-J. Eichhorn,

B. Voit, and F. Kremer, Macromolecules 2010, 43, 9937-9944.

(13) M. Tress, E. U. Mapesa, W. Kossack, W. K. Kipnusu, M. Reiche, and F. Kremer, Science 2013, 341, 1371-1374.

(14) Ed. O.E. Mogensen, Positron annihilation in chemistry, Springer series in chemical physics 58; Springer: Berlin, 1995.

(15) Jean, Y. C., Mallon, P. E., Schrader, D. M. Principles and applications of positron \& positronium chemistry; Eds.;World Scientific: River Edge, NJ, 2003.

(16) Front Matter, in Materials Science of Membranes for Gas and Vapor Separation, Y.

Yampolskii, Pinnau, I. and Freeman, B. (eds.), Editor. 2006, John Wiley \& Sons, Ltd: Chichester, UK.

(17) Harms S., Rätzke K., Faupel F., Chaukura N., Budd P. M., Egger W., Ravelli L., The Journal of Adhesion 2012, 88, 608-619.

(18) Harms S., Rätzke K., Faupel F., Schneider, G. J., Willner, L. and Richter, D. Macromolecules 2010, 43, 10505-10511. 
(19) Matson, S. M., Rätzke, K; Shaikh, M. Q., Litvinova, E. G., Shishatskiy, S. M., Peinemann, K.-V.r; Khotimskiy, V. S., Polymer Science A 2012, 54, 8, 671-677.

(20) Principles and applications of positron \& positronium chemistry; ed. Jean, Y. C., Mallon, P. E., Schrader, D. M., Eds.; World Scientific: River Edge, NJ, 2003.

(21) Nakanishi, H., Wang, S. J. and Jean, Y. C. in Positron Annihilation Studies of Fluids; Sharma S.C., Ed.; World Scientific, Singapore, 1988, 292.

(22) Kruse, J., Kanzow, J., Rätzke, K., Faupel, F., Heuchel, M., Frahn, J., Hofmann, D. Macromolecules 2005, 38, 9638-9643.

(23) Schmidtke, E.; Günther-Schade, K.; Hofmann, D.; Faupel, F., Journal of Molecular Graphics \& Modelling 2004, 22, 309-316.

(24) Sperr, P.; Egger, W., Kögel, G., Dollinger, G., Hugenschmidt, C., Repper, R. et al., Appl Surf Sci. 2008, 255, 35-38.

(25) Harms, S., Rätzke, K., Zaporojtchenko, V., Faupel F., Egger, W., Ravelli, L. Polymer 2011, 52, 505-509.

(26) Algers, J., Sperr, P., Egger, W., Kögel, G., Maurer, F. Phys. Rev. B. 2003, 67, 125404(7).

(27) Coleman, P., Positron beams and their applications, World Scientific, Singapore 2000.

(28) Dryzek, J., Horodek, P., Nuclear Instruments and Methods in Physics Research B 2008, 266, 4000-4009.

(29) Dryzek, J., Horodek, P., Nuclear Instruments and Methods in Physics Research B 2009, 267, 3580-3589.

(30) Allison, J. et al. IEEE Transactions on Nuclear Science 2006, 53, 270-278. 
(31) Kansy, J. J. Nucl. Instrum. Meth. A. 1996, 374, 235-244.

(32) Hess, B., Kutzner, C., van der Spoel, D. and Lindahl, E. J. Chem. Theory Comput. 2008, 4, 435-447.

(33) Lindahl, E., Hess, B. and van der Spoel, D. J. Mol. Model. 2001, 7, 306-317.

(34) Berendsen, H., van der Spoel, D. and van Drunen, R., Comput. Phys. Commun. 1995, 91, 4356.

(35) Johnston K., Harmandaris V., Journal of Physical Chemistry C 2011, 115, 30, 14707-14717.

(36 B. Löwe, private communication.

(37) Depth profiling of the free volume at a Teflon AF/SiN interface with a focused slow positron beam, Ohrt,C.; Koschine,T.; Rätzke,K.; Faupel, F.; Oshima,N; Kobayashi, Y.; Suzuki, R.; Uedono, A (2014) In preparation.

(38) Elsner A., Wagner, A., Aste, T., Hermann, H. and Stoyan, D., Journal of Physical Chemistry B 2009, 113, 7780-7784.

(39 )Schmitz, H. and Müller-Plathe, F., Journal of Chemical Physics 2000, 112, 1040-1045. 


\section{for Table of Contents use only}

\section{INTERPHASE OF A POLYMER AT A SOLID INTERFACE}

H.J. Butt ${ }^{2}$, H. Duran ${ }^{2,5}$, W. Egger ${ }^{6}$, F. Faupel ${ }^{1}$, V. Harmandaris ${ }^{2,3}$, S. Harms ${ }^{1}$, K. Johnston ${ }^{* 2,4}$, K. Kremer ${ }^{2}$, F.Y. Lin ${ }^{2}$, L. Lue ${ }^{4}$, C. Ohrt ${ }^{1}$, K. Raetzke ${ }^{1}$, L. Ravelli ${ }^{6}$, W. Steffen ${ }^{* 2}$, S.D.B. Vianna ${ }^{2}$ 


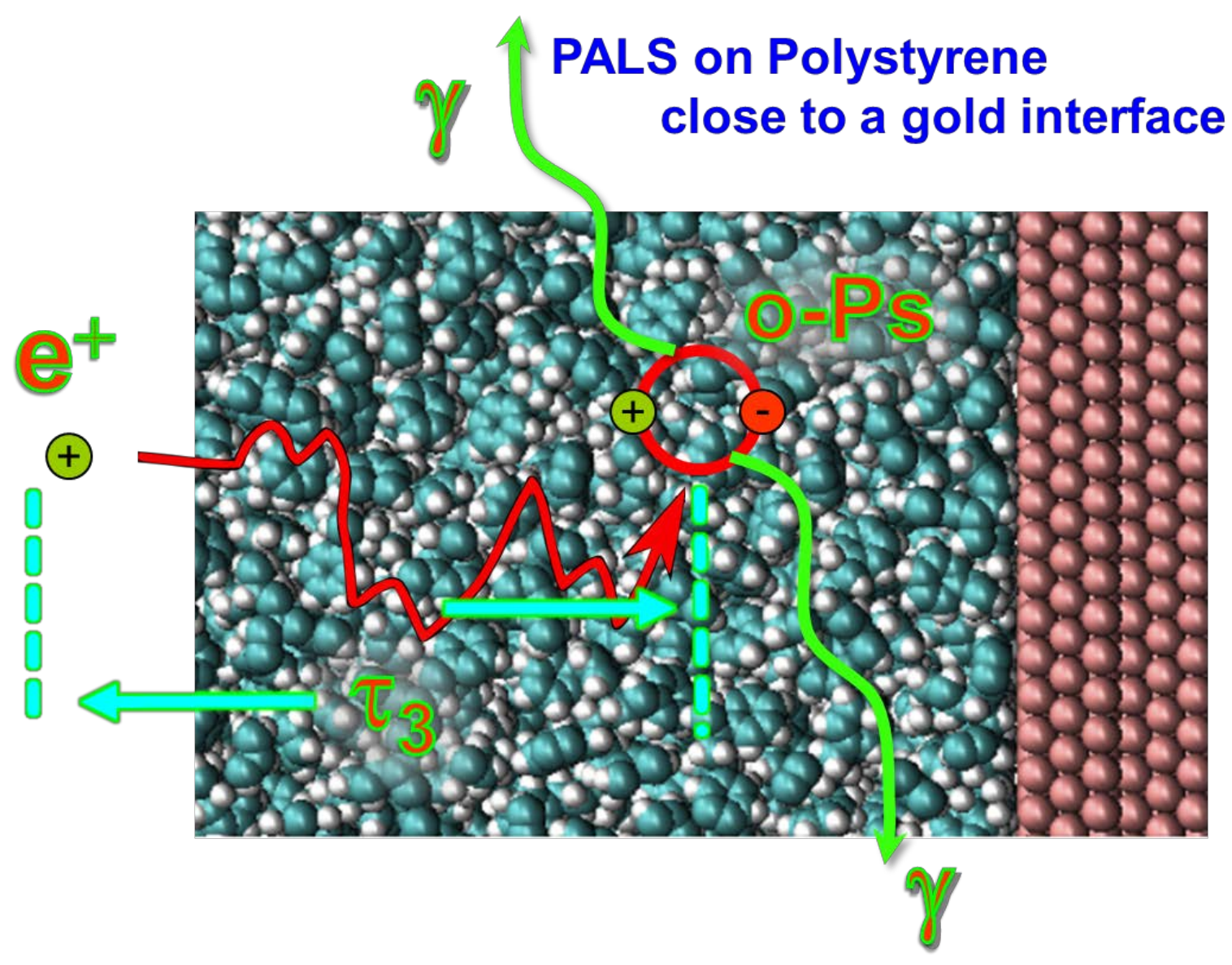

